\title{
Finanzas corporativas en las Empresas de Servicios Públicos de Subachoque y El Rosal Cundinamarca*
}

\author{
Campo Elías López-Rodríguez** \\ Michael David Gil-Muñoz ${ }^{* * *}$ \\ Recibido: 11 de julio de 2020 - Aprobado: 14 de septiembre de 2020 \\ https://doi.org/10.22395/seec.v23n55a1
}

\section{RESUMEN}

Este estudio reconoce la incidencia de las políticas corporativas en la gerencia financiera de las Empresas de Servicios Públicos de Subachoque y El Rosal del departamento de Cundinamarca. A manera reflexiva, sustentada en un enfoque descriptivo, se generó un diagnóstico financiero para identificar los aspectos que influyen en el desarrollo de las políticas corporativas de estas organizaciones, resaltando la incidencia del gobierno corporativo en su desarrollo financiero. Los resultados determinan que estas no incluyen dentro de sus políticas corporativas el apalancamiento financiero de sus operaciones; así mismo, respecto a políticas de inversión, se refleja que están planeando sus presupuestos para cumplir con las obligaciones de funcionamiento, descargando la responsabilidad de generación de valor y crecimiento a las alcaldías municipales.

\section{PALABRAS CLAVE}

Políticas coorporativas, gestión financiera, empresas de servicios públicos, gobierno coorporativo

\section{CLASIFICACIÓN JEL}

G31, H11, H72, L32.

\section{CONTENIDO}

Introducción; 1. Revisión de la literatura; 2. Metodología; 3. Resultados; 4. Conclusiones; Bibliografía.

\footnotetext{
El presente artículo es producto de los procesos de investigación de la sublínea Finanzas Corporativas del programa de Especialización en Gerencia Financiera de la Corporación Universitaria Minuto de Dios - Uniminuto. Producto asociado al Grupo de Investigación Goees con categoría C de Minciencias.

•- Administrador de Empresas, Universidad de Cundinamarca, Fusagasugá, Colombia. Especialista en Gerencia de Mercadeo, Universidad de la Salle, Bogotá, Colombia. Magíster en Gerencia Estratégica de Mercadeo, Politécnico Grancolombiano, Bogotá, Colombia. Líder de Investigación del programa de Especialización en Gerencia Financiera de la Corporación Universitaria Minuto de Dios - Uniminuto. Dirección: Tv. 73a No. 81 G-19, oficina 302 Bogotá, Colombia. Correo electrónico: clopezr3@uniminuto.edu.co. Orcid: https://orcid.org/0000-00034061-2979

*. Contador Público, Corporación Universitaria Minuto de Dios - Uniminuto, Bogotá, Colombia. Especialista en Gerencia Financiera, Corporación Universitaria Minuto de Dios - Uniminuto. Gerente general, Aguas y Aseo de Subachoque SA ESP: Subachoque, Colombia. Dirección: Carrera 2 No. 4-25, oficina 205. Correo electrónico: mgilmuoz@uniminuto.edu.co. Orcid: https://orcid.org/0000-0002-0213-1093
} 


\title{
CORPORATE FINANCE IN PUBLIC SERVICES COMPANIES IN SUBACHOQUE AND EL ROSAL CUNDINAMARCA
}

\begin{abstract}
This study recognizes the incidence of corporate policies in the financial management of the Public Service Companies of Subachoque and El Rosal in the department of Cundinamarca. Reflectively, based on a descriptive approach, a financial diagnosis was generated to identify the aspects that influence the development of the corporate policies of these organizations, highlighting the impact of corporate governance on their financial development. The results determine that these do not include within its corporate policies the financial leverage of its operations; Likewise, regarding investment policies, it is reflected that they are planning their budgets to comply with the operating obligations, releasing the responsibility for value generation and growth to the Municipal Mayors.
\end{abstract}

\section{KEY WORDS}

Corporate policies, financial management, public services, corporate governance.

\section{JEL CLASSIFICATION}

G31, H11, H72, L32.

\section{CONTENT}

Introduction; 1. Review of the literature; 2. Methodology; 3. Results; 4. Conclusions; Bibliography.

\section{FINANCIAMENTO CORPORATIVO NAS EMPRESAS DE SERVIÇOS PÚBLICOS DE SUBACHOQUE E EL ROSAL, CUNDINAMARCA* RESUMO}

Este estudo reconhece o impacto das políticas corporativas na gestão financeira das empresas de serviços públicos Subachoque e El Rosal, no departamento de Cundinamarca. De forma reflexiva, com base em uma abordagem descritiva, foi gerado um diagnóstico financeiro para identificar os aspectos que influenciam o desenvolvimento das políticas corporativas dessas organizações, destacando o impacto da governança corporativa no seu desenvolvimento financeiro. Os resultados determinam que elas não incluem em suas políticas corporativas a alavancagem financeira de suas operações. Da mesma forma, em relação às políticas de investimento, reflete-se que elas estão planejando seus orçamentos para cumprir as obrigações operacionais, cabendo aos governos municipais a responsabilidade pela geração de valor e crescimento.

\section{PALAVRAS-CHAVE}

Finanças; serviços públicos; governança corporativa.

\section{CLASSIFICAÇÃO JEL}

G31, H11, H72, L32.

\section{CONTEÚDO}

Introdução; 1. Revisão da literatura; 2. Metodologia; 3. Resultados; 4. Conclusões; Bibliografia. 


\section{INTRODUCCIÓN}

Unas de las necesidades básicas en el país y en el mundo entero es la de garantizar la calidad de vida a sus poblaciones a través de educación, vivienda, salud, entre otros. Dentro de estos requisitos elementales, se ha destacado la prestación de servicios públicos domiciliarios de agua potable y saneamiento básico, con estándares mínimos de calidad bajo los cuales se puedan satisfacer necesidades prioritarias del ser humano, garantizando la continuidad en los mismos y el cuidado del medio ambiente como principal receptor de las cargas que estos servicios implican.

En Colombia, la responsabilidad de prestar servicios de agua potable y saneamiento básico fue asumida por las autoridades municipales de cada región a través de las secretarias de servicios públicos domiciliarios, vigiladas por la Superintendencia de Servicios Públicos del país con el fin de garantizar la calidad del bien objeto del servicio público, la ampliación permanente del servicio, la continuidad, eficiencia, entre otros fines. Por cumplimiento a la Ley 1176 de 2007 y al artículo 6 de la Ley 142 de 1994, durante la última década, las secretarias de servicios públicos han ido desapareciendo, dándole paso a la creación de las empresas de servicios públicos en cada uno de los municipios, generando de esta manera un independencia política y financiera en el manejo de los recursos destinados para la prestación de los servicios ya descritos con anterioridad.

De ahí la necesidad de realizar un análisis de las condiciones financieras y corporativas de las Empresas de Servicios Públicos (ESP) en los municipios del El Rosal y Subachoque, pertenecientes al departamento de Cundinamarca, e identificar el impacto en el funcionamiento y la prestación de servicios que desarrollan estas entidades, pues se asume que este cambio se llevó a cabo con el fin de obtener la independencia, eficiencia y eficacia en la prestación de los servicios, y, por consiguiente, se deben realizar cambios en la gerencia general, realizando una transición de políticas públicas a políticas corporativas, ante todo en las decisiones relacionadas con la gerencia financiera de cada una de estas empresas, que permitan suscitar unos resultados positivos y de crecimiento tanto para la empresa como para la comunidad beneficiaria (López Rodríguez, Cardozo, Monroy y González, 2019).

En la tabla 1 se refleja la población censada en el año 2005 por el Departamento Administrativo Nacional de Estadística (DANE) y un comparativo con el crecimiento al año 2017 según la población estimada en cada uno de los municipios. Como se observa, los dos municipios tienen un crecimiento similar en el trascurso de 12 años, demostrando un aumento del 29,51 \% y un $34 \%$ para Subachoque y El Rosal, Cundinamarca. 
Tabla 1. Censo poblacional año 2005 y 2017 de los municipios de El Rosal y Subachoque, Cundinamarca.

\begin{tabular}{ccccccc}
\hline Municipio & Área & Viviendas & Hogares & $\begin{array}{c}\text { Personas } \\
2005\end{array}$ & $\begin{array}{c}\text { Personas } \\
2017\end{array}$ & $\begin{array}{c}\text { Incremento } \\
\text { Poblacional }\end{array}$ \\
\hline \multirow{3}{*}{ El Rosal } & Cabecera & 1.438 & 2.614 & 9.736 & & \\
& Resto & 957 & 948 & 3.697 & & \\
& Total & 2.395 & 3.562 & 13.433 & 18.000 & $34,00 \%$ \\
\hline \multirow{5}{*}{ Subachoque } & Cabecera & 1.338 & 1.444 & 5.292 & & \\
& Resto & 2.262 & 2.078 & 7.680 & & \\
& Total & 3.600 & 3.522 & 12.972 & 16.800 & $29,51 \%$ \\
\hline
\end{tabular}

Fuente: Elaboración propia con base en el DANE (2005) y Population.City (2017).

Es importante tener en cuenta este crecimiento poblacional, puesto que, a la par, la demanda para los servicios públicos se verá al alza y por ende cada una de las empresas deberá pensar en una mayor capacidad de cobertura que permita cubrir dicho crecimiento, generado con el paso del tiempo, así como lo demuestra la figura 1 .

Figura 1. Incremento poblacional del año 2005 al año 2017 de los municipios de El Rosal y Subachoque.

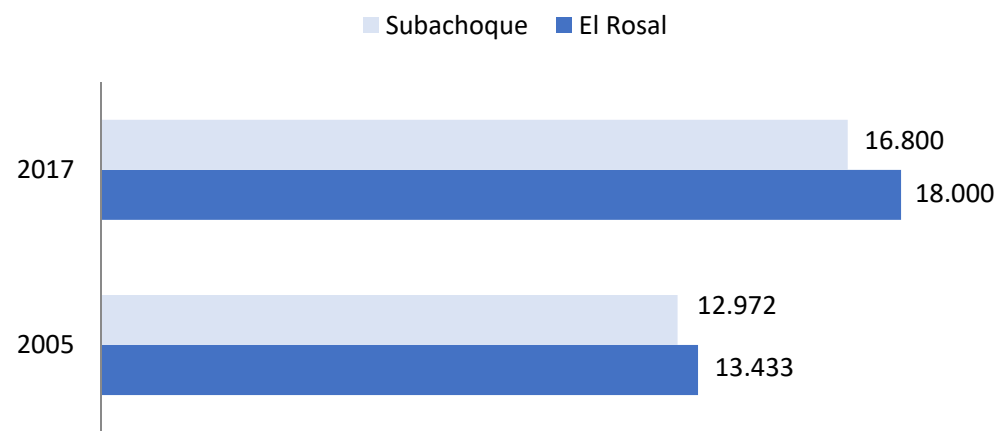

Fuente: Elaboración propia con base en el DANE (2005) y Population.City (2017).

Teniendo en cuenta lo anterior, este estudio pretende dar respuesta a la siguiente formulación del problema: ¿̇cuál es la incidencia de implementar políticas corporativas en la gerencia financiera de las empresas de servicios públicos (SA ESP) de los municipios Subachoque y El Rosal en Cundinamarca? Para ello, sus objetivos radican en relacionar los conceptos de las políticas corporativas y de la gerencia financiera 
en el entorno de los servicios públicos, desarrollar un diagnostico financiero para identificar los aspectos que inciden en el desarrollo de las políticas corporativas de las organizaciones estudiadas, para así, definir la incidencia del gobierno corporativo en el desarrollo financiero de las empresas estudiadas. La literatura revisada sustenta los conceptos de gerencia financiera y de políticas corporativas como plataformas teóricas de la investigación. Esta se desarrolla a partir de un alcance descriptivo de tipo cualitativo por medio de una revisión documental. La población de estudio se concentra en dos empresas de servicios públicos domiciliarios de los municipios estudiados, las cuales poseen características semejantes en su objeto social y en su estructura organizacional.

En principio, el documento presenta una revisión de literatura centrándose en los conceptos de gerencia financiera y de políticas corporativas, luego se relaciona la estrategia metodológica y se establecen los resultados más representativos de la investigación. Para finalizar se proponen las conclusiones del estudio.

\section{REVISIÓN DE LA LITERATURA}

\subsection{Gerencia financiera}

Las entidades con gerencia financiera eficiente no tienen mayores exigencias de rentabilidad por parte de sus inversores, permitiendo de esta manera maximizar sus beneficios económicos a un menor costo de capital (Ferrero, 2014). Así mismo, Gil (2009) menciona que las políticas corporativas y gerencia financiera frente a la estructura de capital en una entidad, son los pilares para encaminar las acciones que promuevan un correcto desarrollo y utilización de los recursos. Para Polanco, Ramírez y Orozco (2016) la experiencia demuestra que las estrategias de las entidades son el reflejo de las decisiones tomadas por los directivos, quienes a través de su liderazgo podrían encaminarlas hacia propuestas globales; por ello, el crecimiento de una entidad se debe basar en fortalecer la creatividad, la motivación, el sentido de pertenencia de quienes la componen, apoyados en establecer mejores competencias y mayor responsabilidad (Pérez, 2016).

Aguilar, Torres y Salazar (2017) aseguran que una gerencia financiera adecuada con el capital de trabajo se refleja en mayor liquidez para cubrir pasivos corrientes, evidenciando un correcto ciclo de transformación del efectivo; de esta manera la gerencia financiera impacta de forma directa la liquidez y rentabilidad de la entidad, lo cual significa que el manejo del capital de trabajo definirá el riesgo asumido y los resultados esperados (Selpa y Espinosa, 2009); por esto, se deben aprovechar los pilares de las entidades como misión, visión y estrategias, para ser 
más llamativos y fortalecer la inclusión de elementos que aporten a los desafíos de sostenibilidad de la entidad (Contreras y Rojas, 2015).

Vázquez (2001) afirma que la internacionalización ha generado que la gerencia financiera se vuelva un ejercicio retador en la sostenibilidad de una entidad, dados los márgenes de especulación que se manejan a la hora de tomar una decisión por parte de los directivos; por lo que los gerentes de las entidades deben promover la creación de valor, el cual permitirá ser sostenibles con el tiempo (López Rodríguez, Cadena López, Montenegro Castillo y Plata Niño, 2019).

Auriac (2010) dice que las actividades de las empresas demuestran valor a su entorno a través de sus políticas de sostenibilidad, retroalimentando el desarrollo de los territorios; ya que las personas cada vez más buscan entidades en las cuales valoren el recurso humano, asuman responsabilidades con el entorno y rindan cuentas a la sociedad (De Oliveira Filho y Abadía, 2013).

En una economía que cada vez es más difícil, y donde las decisiones financieras deben ser tomadas con mayor responsabilidad, se evidencia que los estándares de educación financiera son muy bajos (Giraldo-Prieto, et al., 2017); por ello, los gerentes financieros que pretendan la sostenibilidad de su empresa, deberán utilizar herramientas que midan la gestión y los niveles de riesgo asumidos (Gomes, 2018); puesto que, para Gil (2009), estas son importantes ya que ayudan a mejorar la producción y tomar decisiones acertadas, como pueden ser los indicadores de gestión; y se evidencia a través de los resultados de gerencia la importancia que esta tiene para el buen desarrollo de las entidades (Duque, 2014).

Vecino, Rojas y Muñoz (2015) aseveran que la gerencia financiera debe fomentar la inclusión de nuevos proyectos que potencialicen el alcance de mayores excedentes y crecimiento corporativo, pero los gerentes se orientan en la consecución de resultados a corto plazo sin tener en cuenta la sostenibilidad de la entidad, arriesgando la continuidad a largo plazo de estas, sin tener en cuenta que son temas que deben relacionarse entre sí (Baum, et al, 2004). Es por ello, que se debe considerar que una gerencia financiera eficiente permite ser más competitivo a partir del mejor uso de los recursos, alcanzando una mayor rentabilidad desde la disminución de costos, lo que requiere del estudio financiero y económico de la entidad (Navas y Marbelis, 2009).

Hoy en día la eficiencia financiera es el pilar de los inversionistas y los sectores en general, con el fin de conseguir ventajas competitivas para las entidades (Ferrero, 2014); sin embargo, según Pinzón y Osorio (2017) las pequeñas y medianas empresas fundamentan sus actividades en la experiencia administrativa y económica, utilizan su capital sin proyección financiera, no cuentan con organización y suelen ser 
lideradas por una sola persona desde la informalidad económica; es así, como las entidades fracasan en su etapa inicial, en muchos casos debido a la débil gerencia financiera de su capital de trabajo (Aguilar, Torres y Salazar, 2017).

En la gerencia financiera el capital de trabajo es fundamental a la hora de generar valor y resultados sustentables en la entidad, pues este es uno de los pilares para decidir el financiamiento y las acciones operativas (Beschorner, 2014); también se refleja la necesidad de medir los resultados en una entidad como política corporativa, permite establecer las fallas e identificar las buenas decisiones que ayuden a tramitar acciones que favorezcan la entidad a través de los hallazgos encontrados (Pérez, 2016). Para esto, Navas y Marbelis (2009) mencionan que la gerencia financiera, a través de indicadores, es primordial para el uso eficiente de los recursos, la proyección de la entidad, el impulso del sector y la medición de la gestión frente a los rendimientos.

Mutis (2015) manifiesta que cuando se obtengan márgenes positivos, empleando una buena gerencia financiera con su estructura de capital, las entidades podrán utilizar nuevos recursos en una proyección financiera fidedigna, promoviendo el crecimiento de la entidad; para lo cual las estadísticas de producción serán una herramienta básica para la consecución de buenos resultados, ya que ayudan a definir las mejores decisiones de inversión (De la Hoz, Herrera y Gómez, 2014) y el conocimiento que se posea de las nuevas propuestas dadas desde la gerencia financiera, enmarcará los criterios de selección del mismo para lograr las metas puestas por la organización (Vecino, et al., 2015).

Por su parte, para Polanco, Ramírez y Orozco (2016) lo principal es que las entidades entiendan que una gerencia financiera eficiente es fundamental, y deber ser orientada a través de herramientas que ayuden a medir sus resultados e identificar sus fallas; ya que estas entidades tienen mayores beneficios en su reputación, impactando de manera social y económica sus intereses (Ferrero, 2014); buscando metas que se enmarquen en las políticas económicas y sociales (Gil, 2009). Es por ello que Duque (2014) destaca que una gerencia financiera bien pensada conlleva privilegios en el día a día que demuestra sus resultados en los estados financieros a corto plazo.

\subsection{POLÍTICAS CORPORATIVAS}

Desde los años 90, el sector privado presta servicios públicos domiciliarios al ver que tenían competencia para hacerlo (Betancur y Agudelo, 2007); de ahí que Sayago y Sayago (2010, p.94) sostienen que "el agua como activo económico es un recurso 
no biológico renovable, que debe ser gestionado de forma eficiente y de una manera sostenible"; de manera que las organizaciones, que promuevan políticas corporativas eficientes frente al medio ambiente y la parte social, serán tan rentables como las demás e incluyen beneficios por sus acciones (Molina y Clemente, 2010); para ello el gobierno impuso condiciones para que los servicios públicos domiciliarios se prestaran como cualquier otro servicio, incurriendo en inversión y generando rentabilidad sobre la misma (Boehm, 2005).

Según Contreras y Rojas (2015) las ideas corporativas que involucren acciones para cuidar el medio ambiente, a los empleados y a los beneficios financieros de la entidad, son ideas que hacen parte primordial de políticas corporativas sustentables para las entidades. Por su parte, Ferrero (2014) menciona que la responsabilidad social corporativa impacta los costos de capital y la imagen corporativa, puesto que estas prácticas sociales y medioambientales son vistas por el entorno y repercuten en factores económicos y financieros; de esta manera, las organizaciones se deben conducir de manera articulada, con los contratistas y con todo su entorno de interés (Polanco, Ramírez y Orozco, 2016).

En este sentido, Duque (2014) afirma que los cambios organizacionales tienen varias etapas, las cuales deben interpretarse correctamente si se pretende actuar de forma acertada frente a las acciones requeridas, y de esta forma generar valor a la entidad. Se debe tener presente que los cambios en las entidades se dan por presión y no por entendimiento, como afirman Mantere, Schildt y Sillince (2012), ocasionando que la no existencia de metas claras y bien establecidas indique un mayor riesgo en la consecución de resultados. Es allí, donde la educación financiera de un gerente será determinante para una entidad (Gil, 2009).

Según Pinzón y Osorio (2017) las entidades, como agentes económicos en la sociedad, tienen responsabilidades primordiales, ya que son las encargadas de generar empleo, riqueza, cambios en el mercado, cambios en las personas y progreso en una nación; entonces, cuando se realizan acciones encaminadas a generar valor a las entidades, se vislumbra una perspectiva financiera, puesto que priorizan temas amarrados con ingresos y costos (Duque, 2014). Aunque, adicionalmente, se debe tener el instinto de iniciar proyectos que aporten al medio ambiente y a la población, lo cual, es congruente con la intención de obtener beneficios económicos para las empresas (Contreras y Rojas, 2015).

El desarrollo de la misión y visión en una entidad se identifica a través de su cultura organizacional, puesto que allí se evidencian valores, creencias, actitudes y formas de liderar las compañías (Pérez, 2016); de ahí la importancia de generar 
ambientes agradables y del gusto de los empleados, inmersos en actividades organizacionales que incluyan beneficios que promuevan la tendencia hacia los resultados positivos para la entidad (Corbett, 2018). Según Herrera (2015) se ha demostrado que la motivación es uno de los factores que más incide en una entidad, a diferencia de otros aspectos que pretenden generar valor a esta; puesto que, la sostenibilidad se debe identificar desde sus políticas corporativas, donde se evidencie el compromiso empresarial que promueve la generación de dicho valor (Melamed-Varela, Blanco-Ariza y Rodríguez-Calderón, 2018).

Para De Oliveira Filho y Abadía (2013) las políticas corporativas son la unión de principios, procedimientos, estándares y normas que se crean para encaminar la dirección y control de una empresa de forma responsable, equilibrada y ética; teniendo en cuenta esto, De los Ríos Berjillos, Lozano, Valencia \& Ruiz (2012) afirman que las empresas están reestructurando sus políticas corporativas teniendo en cuenta las tendencias de escasez de recursos hídricos, calentamiento global, conciencia social y conflictos culturales; pues, las decisiones acertadas frente a los riesgos en un sector, se basan en los derivados financieros y reclaman gerentes asertivos y eficientes que promuevan el valor de las entidades, en especial en momentos de crisis financiera (Giraldo-Prieto, et al., 2017).

\section{METODOLOGÍA}

El presente proceso investigativo tiene un enfoque metodológico cualitativo, sustentado en información y documentación recopilada a través de los sitios web oficiales de las empresas foco de estudio, entidades de control como la Superintendencia de Servicios Públicos y la Contraloría General de la Nación, lo que permite analizar las diferentes variables que determinan las decisiones corporativas que impactan las finanzas de cada una de las entidades.

El alcance es de tipo descriptivo, toda vez que se espera analizar información de dos empresas de servicios públicos domiciliarios con características semejantes, en las cuales se pretende hallar fenómenos o comportamientos que permitan vislumbrar consecuencias en sus resultados económicos e identificar cuáles variables de tipo corporativo impactan de manera positiva o negativa los intereses financieros de cada una de ellas, y, de esta manera, entender las posibilidades u opciones más favorables para un gerente o administrador al momento de tomar decisiones en empresas de este sector.

La técnica utilizada fue el análisis documental, que para Dulzaides y Molina (2004) es un conjunto de operaciones intelectuales, cuyo principal objetivo es el 
describir los documentos de forma unificada y sistemática para facilitar su recuperación. Para el desarrollo de esta técnica, se llevó a cabo la búsqueda bibliográfica en bases de datos académicas suscritas al Sistema Nacional de Bibliotecas Rafael García-Herreros (http://biblioteca.uniminuto.edu/). Estas bases de datos fueron las siguientes: Scopus, ScienceDirect y EbscoHost. Así mismo, se utilizaron bases de datos de libre acceso como la de Google y se revisaron informes institucionales y gubernamentales que tuvieran información pertinente al alcance de los objetivos propuestos en el presente estudio.

\section{RESULTADOS}

En la composición del balance de cada una de las organizaciones estudiadas, se observan diferentes comportamientos evidenciados en la figura 2, correspondiente al municipio de Subachoque. En al año 2016 el valor de su pasivo reflejaba obligaciones correspondientes a su operación y no contaba con apalancamiento financiero; en este periodo, el total del pasivo concernía al 37,51 \% de sus activos, mientras que en los años 2017 y 2018 se refleja un aumento considerable de endeudamiento, puesto que sus pasivos llegaron a representar más del $60 \%$ de valor total de su activo, lo cual obedece a recursos que la entidad recibió en administración por parte de la Alcaldía Municipal de Subachoque; estos alcanzaban un porcentaje del 74 \% para el año 2017 y $47 \%$ para el año 2018 del total de sus pasivos. Estos recursos fueron recibidos con el fin de realizar inversiones por parte de la entidad en proyectos productivos que beneficien la operación de la empresa. En el año 2019 se cierra con un endeudamiento del 38,61 \%, correspondiente a obligaciones producto de su actividad.

Con estas cifras se puede determinar que la empresa, en sus políticas corporativas, no incluye el apalancamiento financiero como una alternativa de operación que le permita realizar inversiones o proyectos que aporten al desarrollo de la entidad y admita una estructura de capital menos inclinada hacia el riesgo de los accionistas, contemplando costo-beneficio de adquisición de la deuda; por el contrario, la entidad refleja una dependencia de la administración municipal para realizar inversiones, supeditada a la asignación de recursos en administración sustentados en el presupuesto del Estado (Zambrano y Cuchimaque, 2014), sin reconocer si estos generan valor o no a la empresa. 
Figura 2. Composición del Balance del año 2016 al año 2019 de la empresa de servicios públicos (S.A. E.S.P.) del municipio de Subachoque expresado en porcentaje.

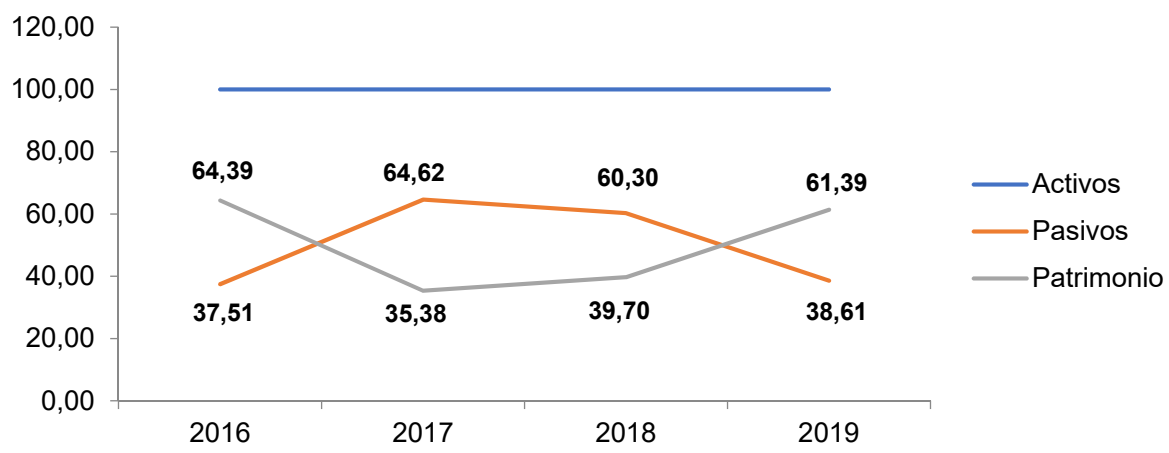

Fuente: Elaboración propia con base en la Contaduría General de la Nación (2019).

Un caso similar es la organización estudiada del municipio de El Rosal, representada en la figura 3, que en el año 2016 se encontraba con una alta concentración del pasivo en la composición del balance, ya que este representaba el $84,98 \%$, es decir, su patrimonio apenas contaba con el 15,02 \% del valor total del activo, pero al igual que la empresa del municipio de Subachoque, contaba con recursos recibidos en administración que correspondían al 65 \% de su pasivo total. Estos recursos fueron teniendo menor peso, hasta llegar a una participación del pasivo dentro de la estructura de capital de un 36,01 \%, lo que genera mayor representación del patrimonio y demuestra que la gerencia financiera determina políticas corporativas de endeudamiento similares a las de la empresa del municipio de Subachoque.

Figura 3. Composición del Balance del año 2016 al año 2019 de la empresa de servicios públicos (S.A. E.S.P.) del municipio de El Rosal expresado en porcentaje.

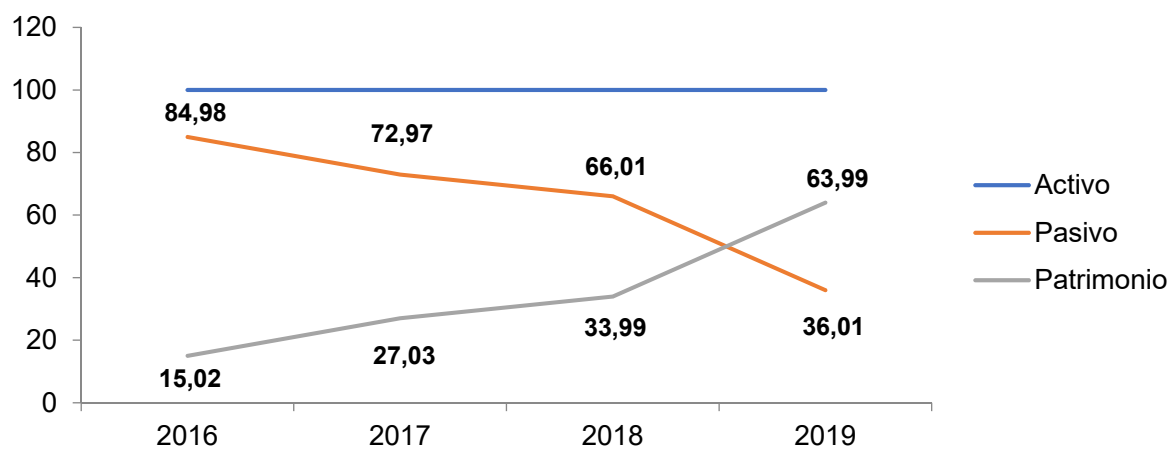

Fuente: Elaboración propia con base en la Contaduría General de la Nación (2019). 
La figura 4 refleja la composición del activo para el año 2019 de las dos empresas, en estese observa una mejor liquidez a corto plazo para la empresa de Subachoque, puesto que el efectivo representa el 50,94 \% en comparación con la empresa de El Rosal, a la cual su efectivo representa apenas el 8,57 \% y su mayor participación en el activo está en propiedades como la planta y equipos con un 46,52 \%; sin embargo, al sumar disponible más inmediato (efectivo y cuentas por pagar) de la empresa de El Rosal, arroja un resultado de $\$ 558.976$ con un pasivo total de $\$ 558.376$, mientras que la empresa de Subachoque tiene $\$ 926.408$ y \$480.146 respectivamente (Contaduría General de la Nación, 2019).

Figura 4. Composición del activo del año 2019 de las empresas de servicios públicos (S.A. E.S.P.) de los municipios de Subachoque y El Rosal expresado en porcentaje

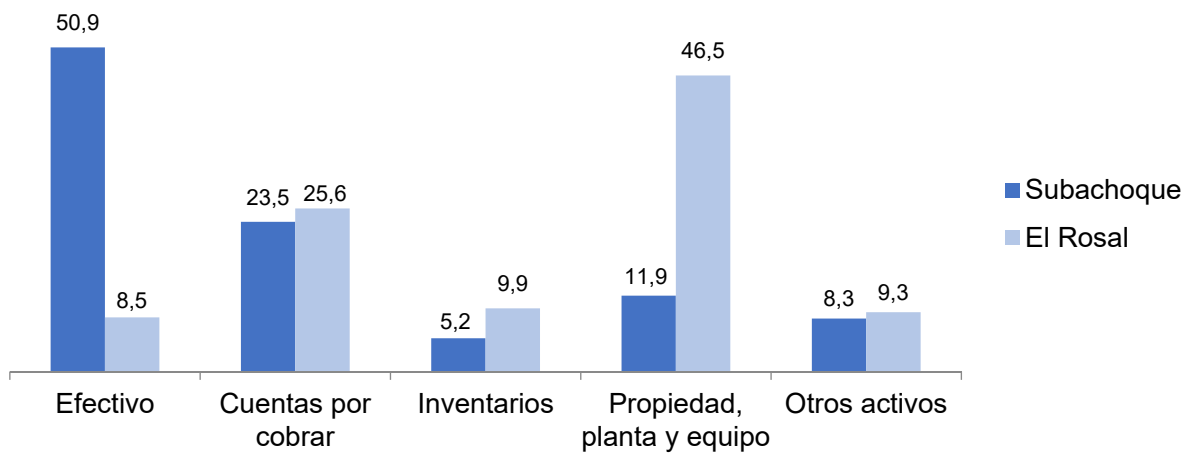

Fuente: Elaboración propia con base en la Contaduría General de la Nación (2019).

Por lo anterior, se podría deducir que la empresa del municipio de El Rosal, en sus políticas corporativas de liquidez, garantiza recursos para cubrir las obligaciones de la entidad, asegurando el cumplimiento de sus obligaciones y dando prioridad a la colocación de recursos propiedad, planta y equipo como políticas de inversión y crecimiento de infraestructura, dando valor a sus activos (Pinzón y Osorio, 2017). Por el contrario, la empresa del municipio de Subachoque refleja un mayor interés por la liquidez de la entidad dentro de sus políticas corporativas y como sustento de su capital de trabajo (Aguilar, Torres y Salazar, 2017).

En la composición del pasivo de la figura 5, se evidencia lo antes mencionado, las dos empresas tienen una política de endeudamiento similar al reflejar una composición equilibrada de acuerdo con el valor total del activo, y en donde se puede percibir que el apalancamiento financiero no existe dentro de sus política e intereses, sus obligaciones las componen las cuentas por pagar obtenidas en el desarrollo de sus actividades y las obligaciones laborales, sin evidenciar una 
sustitución de obligaciones corrientes por obligaciones no corrientes (Zambrano y Cuchimaque, 2014).

Figura 5. Composición del pasivo frente al valor total del activo del año 2019 de las empresas de servicios públicos (S.A. E.S.P.) de los municipios de Subachoque y El Rosal expresado en porcentaje.

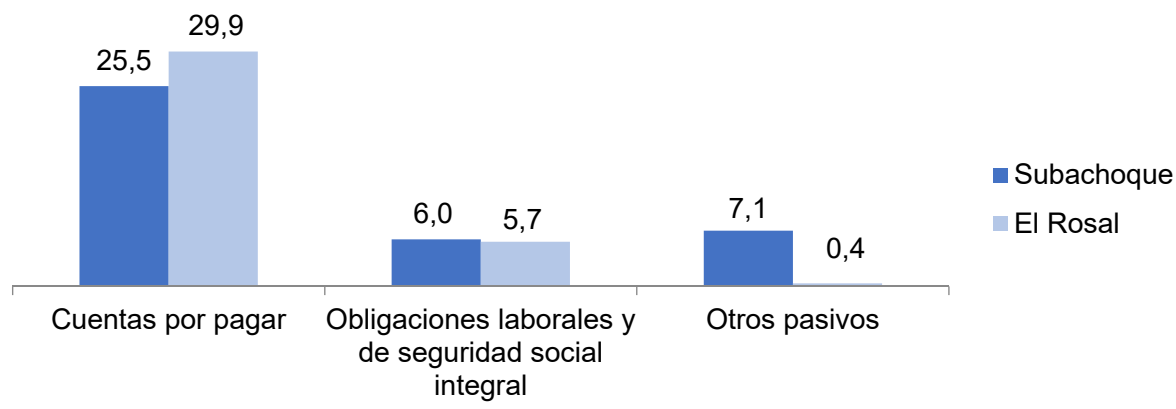

Fuente: Elaboración propia con base en la Contaduría General de la Nación (2019).

En el análisis a los estados de resultados de las empresas presentado en las figuras 6,7 y 8 , se evidencian mejoras en las políticas corporativas y gerencia financiera en cada una de las entidades, pues se refleja un crecimiento considerable en la vigencia 2019, puesto que en sus ingresos se ve un aumento importante, el cual obedece a que durante este periodo se aplicaron tarifas autorizadas por la Comisión de Regulación de Agua Potable y Saneamiento Básico (CRA), mediante Resolución CRA 825 de 2017 adicionada por la resolución CRA 844 de 2018 a nivel nacional y permite tener mejores márgenes a las entidades afectadas.

Esto demuestra que las empresas analizadas no contaban con políticas corporativas que promovieran el crecimiento, expansión y mejora continua de la entidad (Vázquez, 2001; Mutis, 2015; Auriac, 2010; Vecino, et al.,2015); puesto que en las vigencias 2016, 2017 y 2018 más del 97 \% de sus ingresos fueron utilizados para cubrir sus costos y gastos, indicando que sus objetivos se soportan más en el funcionamiento que en el crecimiento como entidad, pues no se evidencia en sus bases de datos ningún crecimiento en la cantidad de usuarios que se pueda atribuir a una expansión de sus servicios.

En las figuras 6 y 8 la empresa del municipio de Subachoque, refleja una política de austeridad en el gasto que financieramente demuestra eficiencia en la ejecución de los ingresos percibidos, mientras que la empresa del municipio de El Rosal vislumbra un crecimiento de sus gastos y costos constante y proporcional entre sí. 
Figura 6. Comportamiento del estado de resultados del año 2016 al 2019 de la empresa de servicios públicos (S.A. E.S.P.) del municipio de Subachoque.

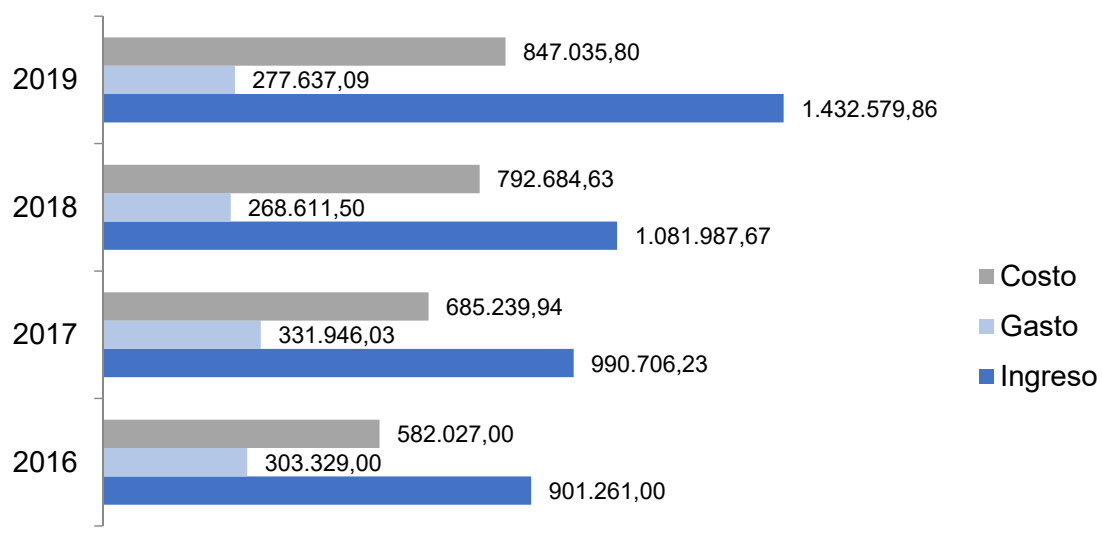

Fuente: Elaboración propia con base en la Contaduría General de la Nación (2019).

Figura 7. Comportamiento del estado de resultados del año 2016 al 2019 de la empresa de servicios públicos (S.A. E.S.P.) del municipio de El Rosal.

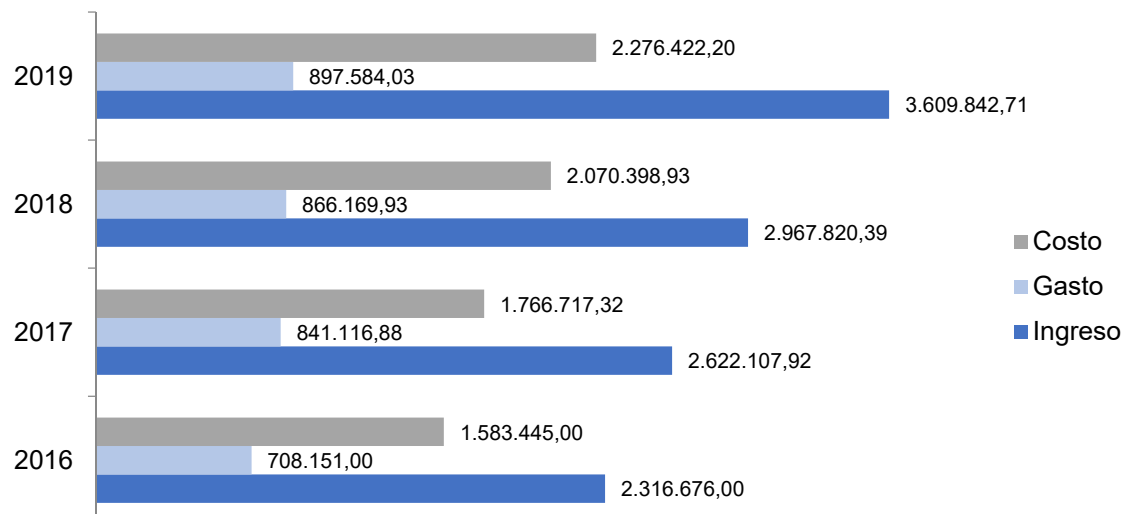

Fuente: Elaboración propia con base en la Contaduría General de la Nación (2019). 
Figura 8. Análisis horizontal de comportamiento de la situación financiera del año 2016 al 2019 de las empresas de servicios públicos (S.A. E.S.P.) de los municipios de Subachoque y El Rosal expresado en porcentaje.

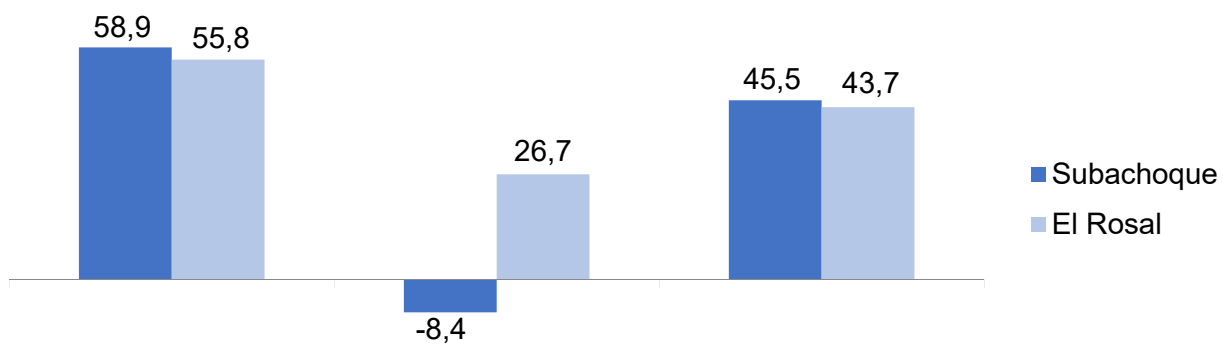

Ingreso Gasto Costo

Fuente: Elaboración propia con base en la Contaduría General de la Nación (2019).

En la figura 9 se puede ver expresada la eficiencia relativa de las empresas de servicios públicos de los municipios de Subachoque y El Rosal. En este se ve representado los márgenes que se han obtenido desde la creación de las dos empresas hasta el año 2019, estas entidades tienen una misma edad y cuentan con un capital suscrito y pagado de $\$ 460.271 .000$ y $\$ 475.910 .000$ para Subachoque y El Rosal respectivamente.

Figura 9. Análisis histórico del (ROE) rendimiento del patrimonio de las empresas de servicios públicos (S.A. E.S.P.) de los municipios de Subachoque y El Rosal expresado en porcentaje.

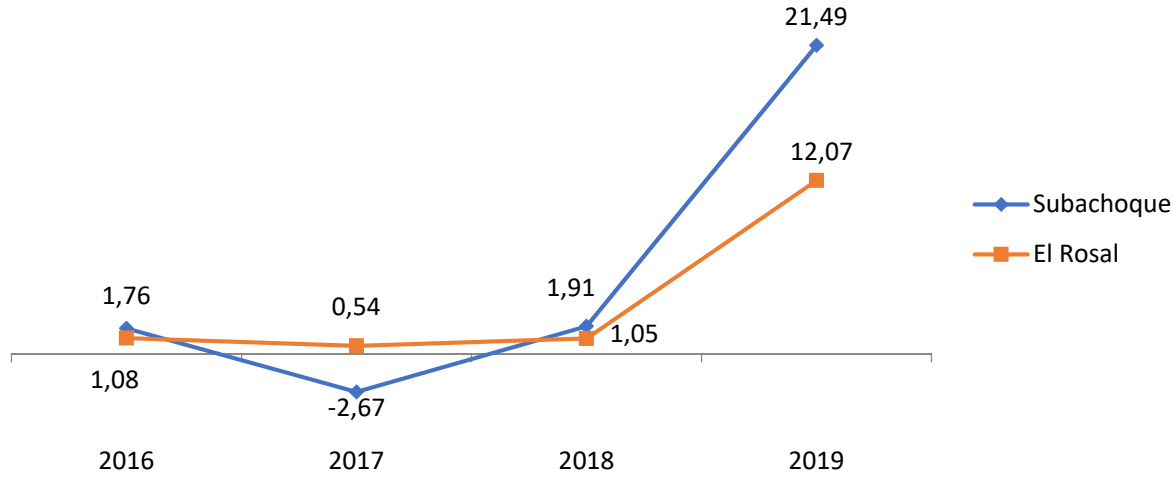

Fuente: Elaboración propia con base en la Contaduría General de la Nación (2019).

Por su parte, la figura 10 permite ver que la eficiencia de los ingresos es muy similar en la operación de las dos entidades, ya que como se ha mencionado con 
anterioridad, demuestra que solo hasta el año 2019 las empresas cuentan con tarifas que generan excedentes a utilizar para encaminar políticas de inversión que permitan mejorar sus resultados, los intereses de los accionistas, la calidad del servicios y cobertura (Boehm, 2005; Betancur y Agudelo, 2007).

Figura 10. Análisis del porcentaje de margen neto histórico en las empresas de servicios públicos (S.A. E.S.P.) de los municipios de Subachoque y El Rosal.

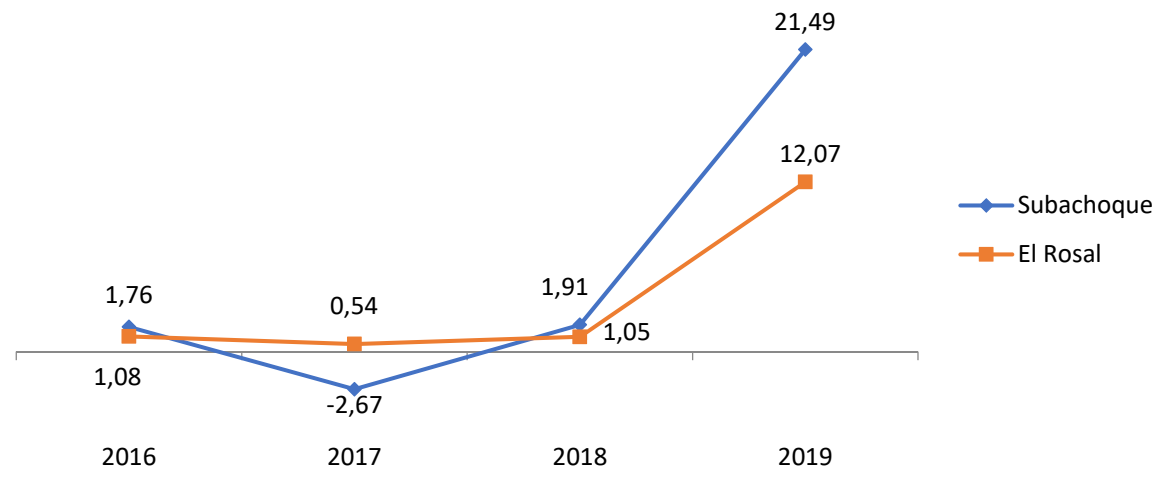

Fuente: Elaboración propia con base en la Contaduría General de la Nación (2019).

\section{CONCLUSIONES}

Algunos de los referentes consultados coinciden en ponderar el crecimiento, sostenibilidad, desarrollo y generación de valor, como uno de los pilares dentro de las políticas corporativas de una entidad, asegurando que la eficiencia de los recursos se promueve por medio de nuevos proyectos e inversiones que garanticen las sostenibilidad en el tiempo y demuestren fortalecimiento a los intereses, no solo de sus accionistas, sino de sus clientes y potenciales demandantes (Navas y Marbelis, 2009; Contreras y Rojas, 2015; Melamed-Varela, et al., 2018; Briozzo Albanese y Santolíquido, 2017).

Otra de las políticas que se pueden resaltar es la relevancia que tiene la liquidez de una compañía al momento de mitigar riesgos de impago y cumplimiento de sus obligaciones, por lo cual se debe garantizar que los pasivos corrientes de una entidad tengan la suficiente cobertura por parte de sus activos corrientes y así definir un capital de trabajo con solidez que establezca garantías y admita aprovechar oportunidades de mejora (Tesfa y Chawla, 2018; Selpa y Espinosa, 2009).

Pérez (2016), De Oliveira Filho y Abadía (2013), Contreras y Rojas (2015) y Pinzón y Osorio (2017) mencionan la importancia que tiene la educación financiera de los 
empresarios, así como la experiencia y motivación del potencial humano, factores que se verán reflejados de forma directa en los estados financieros al afectar la productividad en la toma de decisiones y en la asertividad de las mismas. Esta educación y competencias en los empresarios que tienen participación en las disposiciones finales de las entidades, deberán estar acompañadas de herramientas que sirvan de insumos en las medidas adoptadas, como los indicadores financieros, de gestión y otros, que ayuden a tener un panorama más amplio y fidedigno que permita ser más objetivo al optar por un direccionamiento organizacional a nivel financiero (Giraldo-Prieto, et al., 2017; Gil, 2009; De la Hoz, et al., 2014).

El servicio público de acueducto es muy favorecido al contar con recursos renovables, por ello se debe gestionar muy bien su aporte al medio ambiente y las inversiones que se realizan para que estas generen rentabilidad como cualquier otra industria (Sayago y Sayago, 2010; Molina y Clemente, 2010; Betancur y Agudelo, 2007). Es por ello que las políticas corporativas de estas entidades deben tener un espacio amplio en la inversión medioambiental, debido a que estas tendrán repercusiones significativas en su imagen corporativa y, sobre todo, en aspectos financieros al involucrar todo el entorno en sus acciones desarrolladas (Contreras y Rojas, 2015; Ferrero, 2014; López Rodríguez, 2016).

Las empresas analizadas no incluyen dentro de sus políticas corporativas el apalancamiento financiero de sus operaciones, de esta forma se evidencia que su estructura de capital está enfocada a la utilización de recursos propios y de los accionistas, contemplando únicamente pasivos producto de sus compromisos del día a día operativo, sin financiación a largo plazo, ya que estos se encuentran en su mayoría en el pasivo corriente.

El comportamiento del pasivo de las empresas de servicios públicos investigadas, demuestra que la gerencia general contempla, en sus políticas corporativas, la priorización del cumplimiento de obligaciones con sus usuarios y entes reguladores, esperando que la alcaldía municipal sea quien genere proyectos que permitan acceder a recursos con los cuales sea posible realizar inversiones en la entidad, y que, por las características de estos, se desconoce la generación de valor que pueda tener para el crecimiento y proyección de la entidad.

Las políticas de gerencia financiera deberán siempre fomentar y promover nuevas inversiones que forjen mejores excedentes para garantizar la sostenibilidad de la empresa a largo plazo, haciendo estudios rigurosos de las necesidades y capacidades a nivel económico (Vecino et al, 2015; Baum et al., 2004; Navas y Marbelis, 2009). 
En la evolución de los estados de resultados de las dos empresas, se evidencia la carencia de políticas corporativas financieras que fomenten y se preocupen por la generación de valor de las entidades hacia su proyección a futuro, toda vez que las cifras proyectadas dan por entendido que el objetivo es garantizar el funcionamiento de estas, sin desgastar mayores esfuerzos y recursos que generen un cambio o fortalecimiento en el mercado.

Zambrano y Cuchimaque (2014) afirman que las grandes diferencias que se deben presentar entre los prestadores públicos y los privados, es que estos últimos deben propiciar un desarrollo por medio de sus políticas de inversiones para la sostenibilidad en el mercado y la idoneidad al momento de prestar sus servicios; sin embargo, durante el planteamiento del problema al inicio de esta investigación, se postuló la tesis de una conversión de políticas públicas a políticas corporativas en el funcionamiento de las entidades encargadas de prestar servicios de acueducto, alcantarillado y aseo en los municipios de Subachoque y El Rosal, pero, gracias al diagnóstico financiero, evidenciado en los resultados a través de los estados financieros de estas dos empresas, se puede concluir que la independencia, eficiencia y eficacia que se asumía como objetivo de la transformación jurídica de estos prestadores de servicios, no se ha presentado, toda vez que su funcionamiento refleja poca generación de valor a estas entidades y por el contrario, se percibe una dependencia aun de las alcaldías municipales para emprender inversiones que favorezcan los intereses de las empresas y aporten a su proyección como entidad.

Dentro de las principales políticas corporativas financieras se puede resaltar la política de liquidez, en este caso las dos empresas demuestran su preocupación por tener buenos índices y no afectar el cumplimiento de sus obligaciones, garantizando de esta manera la buena relación con sus proveedores y acreedores que repercutan en una imagen positiva para la entidad y en mayores beneficios económicos que se puedan obtener gracias a la oportunidad de sus pagos.

En cuanto a políticas de inversión, este estudio refleja que las compañías están planeando sus presupuestos con el fin de cumplir a cabalidad con las obligaciones inherentes a su funcionamiento y están descargando la responsabilidad de emprender proyectos que generen valor y crecimiento a las alcaldías municipales, las cuales incorporan recursos en administración a estas entidades para la ejecución de proyectos que beneficien su funcionamiento. Es por ello por lo que solo hasta la vigencia 2019 las dos entidades demuestran excedentes en sus estados de resultados, los cuales son producto de una regulación nacional de tarifas y no de iniciativas por parte de las entidades que permitan un crecimiento y mejores márgenes de utilidad. 
Las políticas corporativas de apalancamiento en las empresas estudiadas son casi inexistentes, puesto que en las dos entidades se demuestra que no existen obligaciones financieras que se hayan adquirido con algún fin específico y las cuentas por pagar que se reflejan en sus pasivos son de corto plazo. Esto puede generar una mayor responsabilidad a la dirigencia, ya que el $100 \%$ de su capital de trabajo esta apalancado por recursos propios y de sus dirigentes, impidiendo de esta manera explotar una alternativa corporativa de gestión del riesgo que genere mayores rendimientos a la entidad y sus accionistas.

En una entidad, las políticas corporativas de personal inciden mucho en sus finanzas, es por esto que esta investigación refleja cómo la continuidad y sostenibilidad en los procesos puede impactar los estados financieros, pues se observa que a pesar de tener el mismo tiempo en el mercado, la empresa de El Rosal ha demostrado mayor eficiencia en los recursos aportados por sus accionistas frente a la empresa de Subachoque, esto se podría atribuir a los continuos cambios que esta última ha tenido en su personal dirigente, lo cual impide que los ideales, proyectos y procesos tengan el suficiente tiempo para representar cambios eficientes financieramente y por el contrario signifique desgaste administrativos y financieros que impactan el crecimiento y expansión de las entidades.

\section{REFERENCIAS}

Aguilar, J. G., Torres, S. G., y Salazar, A. A. (2017). Importancia de la administración eficiente del capital de trabajo en las Pymes. Ciencia Unemi, 10(23), 30-39.

Auriac, J. (2010). Corporate social innovation. Organisation for Economic Cooperation and Development. The OECD Observer, (279), 32-33.

Baum, C.L., Sarver, T. y Strickland, K. (2004). EVA, MVA and CEO compensation: Further evidence. American Business Review, 22(2), 82-87.

Beschorner, T. (2014). Creating shared value: The one-trick pony approach. Business Ethics Journal Review, 1(17), 106-112. http://doi.org/10.12747/bejr2013.01.17

Betancur, O. R., y Agudelo, G. D. (2007). El papel de la lealtad en la construcción de redes sociales: una propuesta para la gerencia social de empresas de servicios públicos domiciliarios. Estudios Gerenciales, 23(104), 27-46.

Boehm, F. (2005). Corrupción y captura en la regulación de los servicios públicos. Revista de Economía Institucional, 7(13).

Briozzo, A., Albanese, D., y Santolíquido, D. (2017). Gobierno corporativo, financiamiento y género: un estudio de las pymes emisoras de títulos en los mercados de valores argentinos. Contaduría y Administración, 62(2), 339-357. https://doi.org/10.1016/j.cya.2017.01.005 
Contaduría General de la Nación (2019). Información Financiera. http://chip.gov.co/schip_rt/ index.jsf.

Contreras, O., y Rojas, I. (2015). Teletrabajo y sostenibilidad empresarial. Una reflexión desde la gerencia del talento humano en Colombia. Suma de negocios, 6(13), 74-83. https://doi. org/10.1016/j.sumneg.2015.08.006

Corbett, T. P. (2018). Buy-side liquidity risk management best practices. Investment Professionals, 11(1), 207-218.

DANE (2005). Boletín del censo general 2005. https://www.dane.gov.co/files/censo2005/PERFIL_PDF_CG2005/25260T7T000.PDF.

De la Hoz Granadillo, E. J., Herrera, T. J. F. y Gómez, J. M. (2014). Evaluación del comportamiento de los indicadores de productividad y rentabilidad financiera del sector petróleo y gas en Colombia mediante el análisis discriminante. Contaduría y Administración, 59(4), 167-191. https://doi.org/10.1016/S0186-1042(14)70159-7

De Oliveira Filho, M. L. y Abadía, J. M. M. (2013). El desempeño económico financiero y responsabilidad social corporativa Petrobrás versus Repsol. Contaduría y Administración, 58(1), 131-167. https://doi.org/10.1016/S0186-1042(13)71201-4

De los Ríos Berjillos, A., Lozano, M. R., Valencia, P. T. y Ruiz, M. C. (2012). Una aproximación a la relación entre información sobre la responsabilidad social orientada al cliente y la reputación corporativa de las entidades financieras españolas. Cuadernos de Economía y Dirección de la Empresa, 15(3), 130 140. https://doi.org/10.1016/j.cede.2012.01.004

Dulzaides, M. E. y Molina, A. M. (2004). Análisis documental y de información: dos componentes de un mismo proceso. Acimed, 12(2), 1-1.

Duque, J. L. (2014). Los procesos de cambio organizacional y la generación de valor. Estudios gerenciales, 30(131), 162-171. https://doi.org/10.1016/j.estger.2014.04.005

Ferrero, J. M. (2014). Consecuencias de las prácticas de sostenibilidad en el coste de capital y en la reputación corporativa. Revista de contabilidad, 17(2), 153-162. https://doi.org/10.1016/j. rcsar.2013.08.008

Gil, N. D. (2009). Ranking financiero: Herramienta financiera para medir la productividad de las empresas de comercialización de energía eléctrica. Estudios Gerenciales, 25(111), 13-34. https:// doi.org/10.1016/S0123-5923(09)70069-5

Giraldo-Prieto, C. A., Uribe, G. J. G., Bermejo, C. V. y Herrera, D. C. F. (2017). Coberturas financieras con derivados y su incidencia en el valor de mercado en empresas colombianas que cotizan en Bolsa. Contaduría y administración, 62(5), 1553-1571. https://doi.org/10.1016/j. cya.2017.04.008

Gomes, G. (2018). The relation between working capital, companies' profitability and shareholder value creation: evidence from brazilian listed industrial companies. Escola de Economía de São Paulo, 47(1), 1-48.

Herrera, H. V. (2015). Complementariedad de las inversiones a largo plazo y de capital de trabajo ante oportunidades de negocios y consideraciones de liquidez en países latinoamericanos. Estudios Gerenciales, 31(137), 364-372. https://doi.org/10.1016/j.estger.2015.07.004 
López Rodríguez, C. E. (2016). El gerente competitivo y su rol en las mipymes: una perspectiva desde el marketing. Equidad y Desarrollo, 1(25), 209-224. http://dx.doi.org/10.19052/ed.3726

López-Rodríguez, C. E., Cardozo, C. E., Monroy, L. y González, E. G. (2019). El impacto del valor económico agregado (EVA) en la valoración accionaria de las empresas colombianas entre los años 2015 al 2018. Revista Espacios, 40(43).

López Rodríguez, C. E., Cadena López, S., Montenegro Castillo, D. C. y Plata Niño, J. A. (2019). Incidencia de la banca multilateral en las asociaciones público-privadas: una perspectiva en Colombia. Equidad y Desarrollo, 1(33), 235-253. https://doi.org/10.19052/eq.vol1.iss33.12

Mantere, H.A. Schildt, J.A. y Sillince (2012). Reversal of strategic change. Academy of Management Journal, 55(1), 173-196. http://dx.doi.org/10.5465/amj.2008.0045

Melamed-Varela, E., Blanco-Ariza, A. B., y Rodríguez-Calderón, G. (2018). Creación de valor compartido: estado y contribuciones a la sostenibilidad corporativa. Revista Escuela de Administración de Negocios, (85), 37-56. https://doi.org/10.21158/01208160.n85.2018.2047

Molina, M. C. y Clemente, I. M. (2010). El comportamiento financiero de las empresas socialmente responsables. Investigaciones Europeas de Dirección y Economía de la Empresa, 16(2), 15-25. https:// doi.org/10.1016/S1135-2523(12)60109-9

Mutis, G. (2015). Valor compartido, una estrategia empresarial de alto impacto. Semana Sostenible, 114-118.

Navas, R. y Marbelis, A. (2009). Análisis financiero: una herramienta clave para una gestión financiera eficiente. Revista Venezolana de Gerencia, 14(48), 606-628.

Pérez, A. M. (2016). Desarrollo organizacional. Una mirada desde el ámbito académico. Educación médica, 17(1), 3-8. https://doi.org/10.1016/j.edumed.2015.09.002

Pinzón, M. M., y Osorio, M. G. (2017). Factores incidentes para crear valor compartido en las mipymes de Bogotá. Suma de Negocios, 8(18), 96-105. https://doi.org/10.1016/j.sumneg.2017.10.003

Polanco, J., Ramírez, F., y Orozco, M. (2016). Incidencia de estándares internacionales en la sostenibilidad corporativa: una perspectiva de la alta dirección. Estudios gerenciales, 32(139), 181-192. https://doi.org/10.1016/j.estger.2016.05.002

Population.City (2017). Población en Colombia. http://poblacion.population.city/

Sayago, J. A. y Sayago, J. M. (2010). Tasas por utilización del agua ¿̇Instrumento de asignación eficiente del agua o mecanismo de financiación de la gestión ambiental? Estudios Gerenciales, 26(115), 93-115. https://doi.org/10.1016/S0123-5923(10)70114-5

Selpa, A. Y., y Espinosa, D. (2009). La gestión del capital de trabajo como proceso de la gestión financiera operativa. Gestión Joven. Revista de la Agrupación Joven Iberoamericana de Contabilidad y Administración de Empresas, (4), 1-25.

Tesfa, N. T. y Chawla, A. S. (2018). Working capital management practices: a comparative study of Ethiopia with United States, Australia, Canada and Pakistan. IOSR Journal of Economics and Finance, 9(2), 21-35. https://doi.org/10.9790/5933-0902022135

Vázquez, J. (2001). Resultados del actual proceso de cambio en las estructuras de activo y de pasivo empresarial. Investigaciones Europeas de Dirección y Economía de la Empresa, 7(3), 67-90. 
Vecino, C. E., Rojas, S. C., y Munoz, Y. (2015). Prácticas de evaluación financiera de inversiones en Colombia. Estudios Gerenciales, 31(134), 41 49. https://doi.org/10.1016/j.estger.2014.08.002

Zambrano, S. y Cuchimaque, C. C. (2014). Análisis de la estructura de capital para empresas de Servicios Públicos. In Vestigium Ire, 6(1). 